\title{
The Effect of Stress and Depression on Gastrointestinal Diseases: Author's Reply
}

TO THE EDITOR: We appreciate the interest and comments on our paper, which was published in the April 2015 issue of this journal. ${ }^{1}$ Through our study, we showed that emotional stress and depression may be predisposing factors for functional dyspepsia and irritable bowel syndrome. In addition, as far as I know, this is the first study to show that depression may be linked with elevated gastric cancer risk. However, as Celik et $\mathrm{al}^{2}$ pointed out, this study has some limitations.

Celik et $\mathrm{al}^{2}$ suggested in first point that our study did not specify whether the participants used psychotropic medication or exercised regularly. We completely agree that it is important to clarify whether the subjects were treated with psychotropic medication or not. However, if psychiatric symptoms of depressed patients are relieved by use of antidepressant medication, it may also reduce the incidence of functional GI disorder and cancer risk. Therefore the current severity of stress and depression is more important than the use of psychotropic drugs. On the other hand, there is a general belief that physical activity and exercise can reduce vulnerability to stress and may be associated with decreased symptoms of depression. ${ }^{3,4}$ However, we believe that it is inappropriate to exclude patients who do little exercise. Rather, it may lead to selection bias. Nevertheless, if we had reflected the information about the drug use and physical activity in our study, we might have got a more accurate result.

In the second point, Celik et $\mathrm{al}^{2}$ indicated that the menstrual status of female individuals was not specified in this study. As they mentioned, stress and depression levels can show fluctuations during the menstrual cycle and menopausal period. ${ }^{5,6}$ Therefore it can be helpful to analyze the relationship between the menstrual cycle and the level of stress and depression in our study. Unfortunately, however, the information about the menstrual cycle and periods was not included in the investigating items of our questionnaire.

\section{Sang Pyo Lee and In Kyung Sung Department of Internal Medicine, Konkuk University Medical Center, Seoul, Korea}

1. Lee SP, Sung IK, Kim JH, Lee SY, Park HS, Shim CS. The effect of emotional stress and depression on the prevalence of digestive diseases. J Neurogastroenterol Motil 2015;21:273-282.

2. Celik C, Ozdemir B, Oznur T. The effect of stress and depression on gastrointestinal diseases. J Neurogastroenterol Motil 2015;21:452.

3. Byrne A, Byrne DG. The effect of exercise on depression, anxiety and other mood states: a review. J Psychosom Res 1993;37:565-574.

4. Ströhle A. Physical activity, exercise, depression and anxiety disorders. J Neural Transm 2009;116:777-784.

5. Yonkers KA, O'Brien PM, Eriksson E. Premenstrual syndrome. Lancet 2008;371:1200-1210.

6. Bromberger JT, Matthews KA, Schott LL, et al. Depressive symptoms during the menopausal transition: the Study of Women's Health Across the Nation (SWAN). J Affect Disord 2007;103: 267-272.

Conflicts of interest: None. 